\section{The rotational model: a new hypothesis for thylakoid stacking}

\author{
Antonios Castorinis \\ Biologist, Kifissia, Greece
}

\section{Abstract}

The most enigmatic feature of mature thylakoids of Angiosperms is the presence of piles of membranous discs forming the cylindrical structures known as grana. Although some models aim to elucidate their formation, until now the mechanism governing the architecture of thylakoid stacks remains obscure. In this work a new model is presented aiming to explain the way thylakoids stack. In comparison with previous models, this model proposes a dynamic mechanism for the rapid selfassembly of thylakoid stacks and their subsequent disassembly under the influence of a variety of physicochemical factors and is consistent with the evolutionary origin of these membranes and their ontogenetic continuity. The model proposes that, under the influence of attractive electrostatic forces, the membranes come closer in a parallel alignment and the photosystem I//light harvesting complexes migrate laterally forming circular aggregates. Finally the thylakoids rotate around the vertical axis of the superimposed aggregates, under the action of a torque.

\section{Introduction}

Mature thylakoid membranes of green plants and some green algae are differentiated into unstacked or stroma lamellae and stacked or appressed thylakoids or grana. It is generally believed that, in green plants, stacked and unstacked thylakoids form a continuous membrane that encloses a single compartment, the lumen, and that stacked thylakoids are cylindrical structures with a diameter of 300-600 nm. ${ }^{1-3}$ Thylakoids present structural heterogeneity; the main fraction of photosystem II (PSII) and its light harvesting complex (LHCII) reside mainly in the stacked thylakoids, whereas photosystem I (PSI) and its LHCI, as well as the ATP synthase are predominantly concentrated in stroma lamellae. ${ }^{2}$ Under certain conditions, thylakoids can undergo changes from the stacked to the unstacked state. These changes are rapid, dynamic and reversible. In vivo, the degree of membrane stacking may change during acclimation of plants to different light environments, ${ }^{4}$ whereas in vitro can be affected by changes in the ionic milieu, ${ }^{5}$ in the light intensity, etc. $^{6}$

Many factors have been reported to be implicated in grana formation: electrostatic repulsion between thylakoids carrying net negative charges on their surfaces due to the presence of membrane carboxyl groups, cation modulated electrostatic interaction between opposing thylakoids, Van Der Waals attraction, hydration repulsion, steric hindrance and entropy driven attraction. ${ }^{1,7}$ Besides such physicochemical forces, many studies have suggested that LHCII itself is implicated more or less in the stacking of thylakoids. ${ }^{8,9}$ Electrostatic interactions between PSII/LHCII supercomplexes across the stromal gap have been reported to be responsible for grana formation or for maintaining thylakoid appression. ${ }^{2,10,11}$

Since 1962, several quite static models have been proposed to describe the structure of grana and their interconnections with stroma thylakoids of higher plant chloroplasts, reviewed by Mustardy and Garab ${ }^{3}$ and Nevo and colleagues. ${ }^{12}$ The most dominant models are the folded membrane model, the pairwise organization model and the helical model. In the folded membrane model grana are formed by symmetrical invaginations of stroma thylakoids pairs into piles of three discs. ${ }^{13}$ In the pairwise organization model the granum is formed by bifurcations of the stroma lamellae into multiple parallel discs and subsequent bending and fusion of the newly created membranes. ${ }^{14,15}$ In the helical model the stroma membranes are wound around the granum in the form of multiple right handed helices. ${ }^{3}$

The above mentioned models explain neither the molecular basis nor the involvement of the physicochemical forces on the formation of grana and, thus, the mechanism governing the spatial organization of the thylakoid membranes remains obscure. None of these models provides proof for the evolutionary origin of these cylindrical structures or their ontogenetic continuity. Furthermore, none of these models explains the mechanism of the rapid selfassembly of thylakoid stacks and their disassembly, the role of LHCII, the lateral segregation of the two photosystems or the differences in the extent of thylakoid appression and the relation of thylakoid morphology to the shape of chloroplasts. Therefore, a new model is required to explain the formation of grana. To do so, it is necessary to reexamine the formation of grana in the light of evolution, because, as Theodosius Dobzansky wrote, Nothing in Biology makes sense unless seen in the Light of Evolution.

In oxygenic photosynthetic organisms, such as cyanobacteria, algae and green plants, the photosynthetic machinery comprises two types of reaction centers working in series: PSII and PSI. Structural studies show that all reaction centers of those organisms have a common
Correspondence: Antonios Castorinis, 14 Aiginis Street, T.K. 14561 Kifissia, Attica, Greece. Tel/Fax: +30.210 .8070090 .

E-mail: kastorant@yahoo.gr

Key words: Grana; Light harvesting complex; Thylakoid stacking; Torque; Rotational model.

Acknowledgments: The author would like to thank Dr. Joan Argyroudi-Akoyunoglou for helpful discussion, Ms. Konstantina Kastorini for the Figures' development and Dr. Christina-Maria Kastorini and Dr. Georgios Papadakis for their useful comments regarding the manuscript's presentation.

Conflict of interest: the author declares no potential conflict of interest.

Received for publication: 6 October 2015. Revision received: 24 October 2015. Accepted for publication: 24 October 2015.

This work is licensed under a Creative Commons Attribution-NonCommercial 4.0 International License (CC BY-NC 4.0).

CC Copyright A. Castorinis, 2016

Licensee PAGEPress srl, Italy

International Journal of Plant Biology 2016; 7:6237 doi:10.4081/pb.2016.6237

overall structure. ${ }^{16}$ However, the morphology of their photosynthetic membranes presents striking differences. Cyanobacteria (photosynthetic unicellular prokaryotes) as light harvesting antenna possess large protein complexes, the phycobilisomes. In most cyanobacteria, photosynthesis is carried out in 3-10 concentric thylakoids that follow the periphery of the cell. ${ }^{12}$ In Algae, the photosynthetic membranes are enclosed within specialized organelles called chloroplasts. Chloroplasts are derived from endosymbiosis of an ancient cyanobacterium by a eukaryotic host cell. ${ }^{17}$ Chloroplasts of Red Algae usually have parallel thylakoids arranged along the envelope of the chloroplast in equal distances. ${ }^{12}$ Chloroplasts of Brown Algae possess thylakoids organized into stacks of three layers, but these stacks are not equivalent to grana. Chloroplasts of Green Algae present different patterns of thylakoids. Other species belonging to the group of Charophyta, possess thylakoids with well defined grana and stroma lamellae presenting lateral heterogeneity similar of higher plants. ${ }^{12}$ Finally, angiosperms (green plants), in their mature chloroplasts, have thylakoids organized in characteristic cylindrical structures of superimposed discoid membranes, the grana. Since the photosynthetic machinery of cyanobacteria, algae and green plants has been impressively conserved during evolution and given that this machinery is located on 
membranes, it is logical to assume that these membranes must have very similar structures as well, in spite of their striking morphological differences. This assumption poses a direct doubt to the existence of grana in the form of cylindrical structures.

Thylakoid formation has also been investigated during ontogenesis. Angiosperms grown in the dark have etioplasts. Each etioplast contains a spherical semicrystalline structure, the prolamellar body. ${ }^{18}$ Upon illumination, the etioplast is transformed into protochloroplast by a photomorphogenic transition: the prolamellar body is disintegrated and long unconnected tubules appear, extending parallel to each other in the stroma and finally, they fuse with each other, forming parallel aligned thylakoid sheets. Subsequently, the thylakoids seem to overlap, forming paired lamellae. Finally, the protochloroplast differentiates into a mature chloroplast with well-defined grana stacks. ${ }^{19}$ This step of differentiation can be slowed down for days if plants are grown in intermittent Light - Dark Cycles (LDC, 2 minutes light followed by 98 minutes dark). Protochloroplasts have only primary thylakoids and the LHCII is absent whereas after 40 LDC these thylakoids begin to form appressions concomitantly with the appearance of LHCII. ${ }^{8,9}$ When plants are transferred to continuous light, after short preexposure to periodic light, greater amounts of LHCII are formed and the lamellae are differentiated into unstacked and stacked thylakoids. However, after prolonged preexposure to periodic light (86-100LDC), the lamellae form arrays of long parallel loosely appressed membranes; transfer of these leaves to continuous light induces the formation of shorter parallel thylakoids and very few grana. $^{20}$

\section{Materials and Methods}

\section{The rotational model}

After the examination of thylakoids in the light of evolution and ontogenesis, the following evidence has emerged: a) grana are, probably, not cylindrical structures of membranous discs; b) the formation of parallel-aligned thylakoids seems to be an intermediate stage before stacking.

The structure of pea LHCII trimer and its charge distribution on the stromal surface has been presented by Standfuss and colleagues, ${ }^{11}$ who proposed that electrostatic attractive forces between LHCII of opposite membranes play a major role in the cohesion of grana. However, a single PSII/LHCII complex can interact electrostatically with many other neighboring PSI/LHCII complexes at any direction. The following interactions are distinguished: i) transverse interactions between
PSII/LHCII - PSII/LHCII of the same thylakoid across the lumen. These interactions tether the opposite membranes, are responsible for the flat shape and the rigidity of thylakoids and monitor the width of the lumen (Figure 1A); ii) transverse interactions between PSI/LHCII PSII/LHCII of adjacent thylakoids across the stroma. These interactions are responsible for the parallel appearance of adjacent thylakoids and regulate the distance between them. (Figure 1B); iii) lateral interactions between PSII/LHCII - PSII/LHCII within the plane of one membrane. These interactions are responsible for the lateral movement of the complexes and the formation of aggregates. (Figure 1C).

Any model concerning thylakoid stacking should explain all the different patterns of thylakoid conformation observed during evolution, i.e., from the parallel arrangement of lamellae, to the close associations of parallel membranes forming bundles and finally, to the formation of grana. It should also explain their different aspects during photomorphogenesis, i.e., from the parallel alignment, to the presence of appressions and finally, to the sudden appearance of stacks. In addition, it should involve a mechanism with the participation of LHCII and the implication of the physicochemical forces and this mechanism must be rapid and reversible.

In the model presented in this work, the protagonistic roles are given to electrostatic forces between the PSI/LHCII complexes. ${ }^{11}$ Constant roles are distributed to electrostatic repulsion between thylakoids carrying net negative charges on their surfaces ${ }^{7}$ and between negatively charged PSI/LHCI complexes. ${ }^{10}$ Fission and fusion reactions, hydration repul$\operatorname{sion}^{7}$ and steric hindrance ${ }^{7}$ also play significant roles. The torque is the guest star appearing at the final act of thylakoid stacking.

\section{Stacking}

At low salt medium, as already reported by Izawa and Good, ${ }^{5}$ thylakoids form a continuous network of parallel membranes (Figure 2A). Negatively charged membrane carboxyl groups $^{21}$ and negatively charged PSI/LHCI complexes ${ }^{10}$ keep the adjacent thylakoids far apart, due to repulsive electrostatic forces (Figure 2, step 1). Furthermore a uniform lateral distribution of all complexes inside the membrane is also established. ${ }^{10}$

The addition of cations causes electrostatic screening on the surface of thylakoids. ${ }^{21}$ So the adjacent thylakoids approach each other in a parallel way. The PSII/LHCII complexes present both positively and negatively charged domains as proposed by Standfuss and colleagues. ${ }^{11}$ Due to the balanced interactions between the repulsive forces exerted by the negatively charged PSI/LHCI complexes ${ }^{10}$ and the attractive electrostatic forces by the PSII/LHCII complexes, the adjacent mem- branes form bunches of parallel thylakoids (Figure 2, step 2).

Besides the transversal interactions, lateral attractive electrostatic forces are exerted between the PSII/LHCII complexes within the plane of one membrane. The PSI/LHCII complexes can move laterally only when the electrostatic forces that are exerted between them, are strong enough to overcome the resistance of the fluid mosaic. These forces increase either with the decrease of the distance between the complexes, or with the increase of their electric charges. The latter increase as the PSII unit size increases, while the distance between them decreases as their number increases during greening. Both conditions are accomplished in mature chloroplasts of angiosperms. Indeed Kohorn and Yakir ${ }^{22}$ have demonstrated that the major protein component of LHCII can migrate through thylakoids. Due to these attractive forces exerted between the neighboring PSI/LHCII complexes, the latter approach each other and form circular aggregates in both the upper and lower membranes of the same thylakoid. Such circular aggregates have been observed by Kouril and colleagues. ${ }^{23}$ During the lateral diffusion of the PSII/LHCII complexes, some of them may be obstructed by other membrane proteins and may remain entrapped in stroma thylakoids. The negatively charged PSI/LHCI complexes and ATP synthase, which contain extended projections into the aqueous stroma, are excluded from the interior of the aggregates by steric hindrance. ${ }^{7}$ As the aggregates keep growing, they expand the membrane, forming discoid patterns with simultaneous sliming of both ends of the thylakoid. Stronger attractive forces are also exerted transversely between opposing complexes across the lumen, forcing the lumen to contract even more (Figure 2, step 3).

Attractive forces are also exerted on the complexes of all the parallel adjacent thylakoids, forcing the complexes to diffuse laterally and form large discoid aggregates. As the aggregates grow, the distance between the parallel-superimposed thylakoids decreases and the attractive electrostatic forces between the circular aggregates of the adjacent membranes become increasingly stronger, forcing them to come closer. As the neighboring underlying and overlying thylakoids approach each other, the membrane regions remaining outside the aggregates, at both ends of the thylakoid, exert increasingly greater repulsive forces, due to the negatively charged PSI/LHCI complexes. Therefore, a torque is formed, causing the superimposed thylakoids to rotate around the vertical axis of the circular aggregates until a static equilibrium is obtained in a lower energy level (Figure 2, step 4). Indeed, Chow and colleagues $^{10}$ suggested that stroma thylakoids carry a higher net negative electrical charge 
than do stacked thylakoids. Hydration repul$\operatorname{sion}^{7}$ at the partition gap allows the rotation and the simultaneous sliding of the stacked membranes, permitting the alignment of the stacks. Under the rotation, stroma thylakoids interconnecting neighboring grana are disrupted. Very early Izawa and Good ${ }^{5}$ underlined such fragmentations. Indeed, these membranes become weakened, due to the dramatic decrease of PSI/LHCII complexes, which tether the two membranes across the lumen. After the formation of stacks, thylakoids may fuse with each other, establishing again a continuous membranous system. This assumption is supported by the presence of a protein that mediates fusion and affects thylakoid organization in Arabidopsis chloroplasts. ${ }^{24}$

During fusion, bifurcations may be created between the disrupted membranes. Finally, the whole chloroplast obtains an ellipsoid shape as already reported by Izawa and Good. ${ }^{5}$

\section{Unstacking}

When stacked thylakoids (Figure 2, step 4) are washed and re-suspended in low salt solution, the PSII/LHCII complexes become negatively charged and segregate laterally, due to repulsive forces (Figure 2, step 3). As a result, the adjacent thylakoids are repelled away, due to their negative charges. The distance between them is increased (Figure 2, step 2) and their parallel arrangement reappears. Finally, a random distribution of all the complexes is established ${ }^{10}$ (Figure 2, step 1) and the whole chloroplast looks swollen in agreement with the observations by Izawa and Good (Figure 2A). ${ }^{5}$

Given that this model does not follow strict rules, it can explain all the irregularities observed. Thylakoids can rotate randomly either clockwise or anticlockwise. The aggregates can be formed anywhere inside the thylakoid, in the middle or at the edges, giving rise to different configurations. When formed in the middle, the stroma lamellae seem to cross the stack perpendicularly; when formed at the edges, the membranes seem to run from one level of the granum to a different level on the opposite side. In vivo, the stacks are formed by combination of these patterns. Even the number of appressed membranes per granum stack is not predetermined. This model accepts, as already proposed by Horton and colleagues ${ }^{25}$ that the aggregation of the light harvesting complexes in vivo is induced by protonation of the lumen.

\section{Further considerations}

The rotational model is a new model that attempts to elucidate the mechanism of stacking, focusing on the evolutionary and ontogenetic continuity of thylakoids. Thus, it rejects the generally accepted idea that grana consist of cylindrical structures of superimposed dis- coid membranes. In this model, the principal role is given to attractive electrostatic forces exerted between the PSI/LHCII complexes. The main steps for stacking are, firstly, the close approach of the parallel thylakoids and the formation of bundles, secondly, the lateral migration of the PSII/LHCII complexes, thirdly, the formation of circular aggregates and fourthly, the rotation of thylakoids around the vertical axis of the aggregates. In accordance with these steps, the following observations should be mentioned. The formation of long parallel bundles and their coexistence with stacks in phaseolus vulgaris chloroplasts transferred to continuous light after prolonged preexposure to periodic light, ${ }^{20}$ advocates that the formation of bundles is the first step. Kohorn and Yakir ${ }^{22}$ have demonstrated that the radiolabeled major protein component of LHCII can migrate through thylakoid membranes, showing that the second step (the lateral migration) is possible. The third step, the formation of circular aggregates, has been demonstrated by Kouril and colleagues, ${ }^{23}$ who observed superimposed circular aggregates consisting of PSI/LHCII complexes in granal thylakoid membranes from spinach, using cryo electron

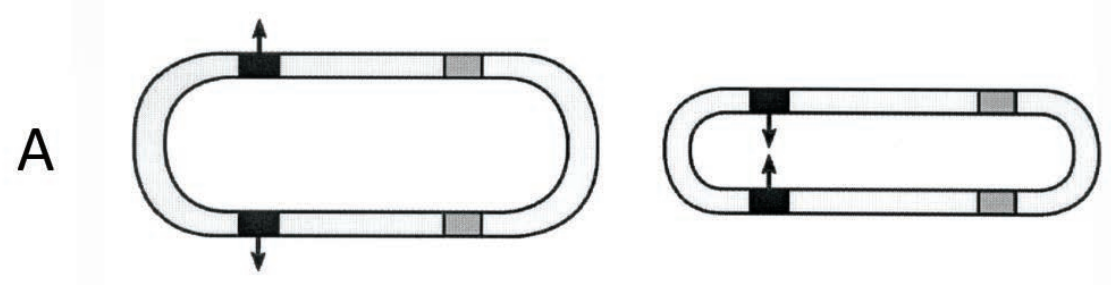

B

Some experimental data can also be explained using the rotational model. It has been reported that plants grown in PSI light have a greater PSII/PSI ratio and a greater abundance of LHCII complexes, while they exhibit a greater extent of granal stacking and smaller non-appressed membrane domains, as compared to plants grown in PSII light. ${ }^{10}$ The

tomography. The forth step, i.e., the rotation of observed directly using electron microscopy. However, the consequences of the rotation, namely the extensive breakage of the memGood, ${ }^{5}$ who noticed a considerable breakage of lamellae between grana. A prerequisite for rotation to occur is that unstacked thylakoids stacked membranes. Indeed it has been proposed that stroma thylakoids carry a higher negative surface charge density than the surbranes. ${ }^{10}$ This model predicts that the width of each superimposed thylakoid sheet is about $300-600 \mathrm{~nm}$. As the thylakoids rotate they form a granum, which seems to have a phenomenal diameter of 300-600 nm
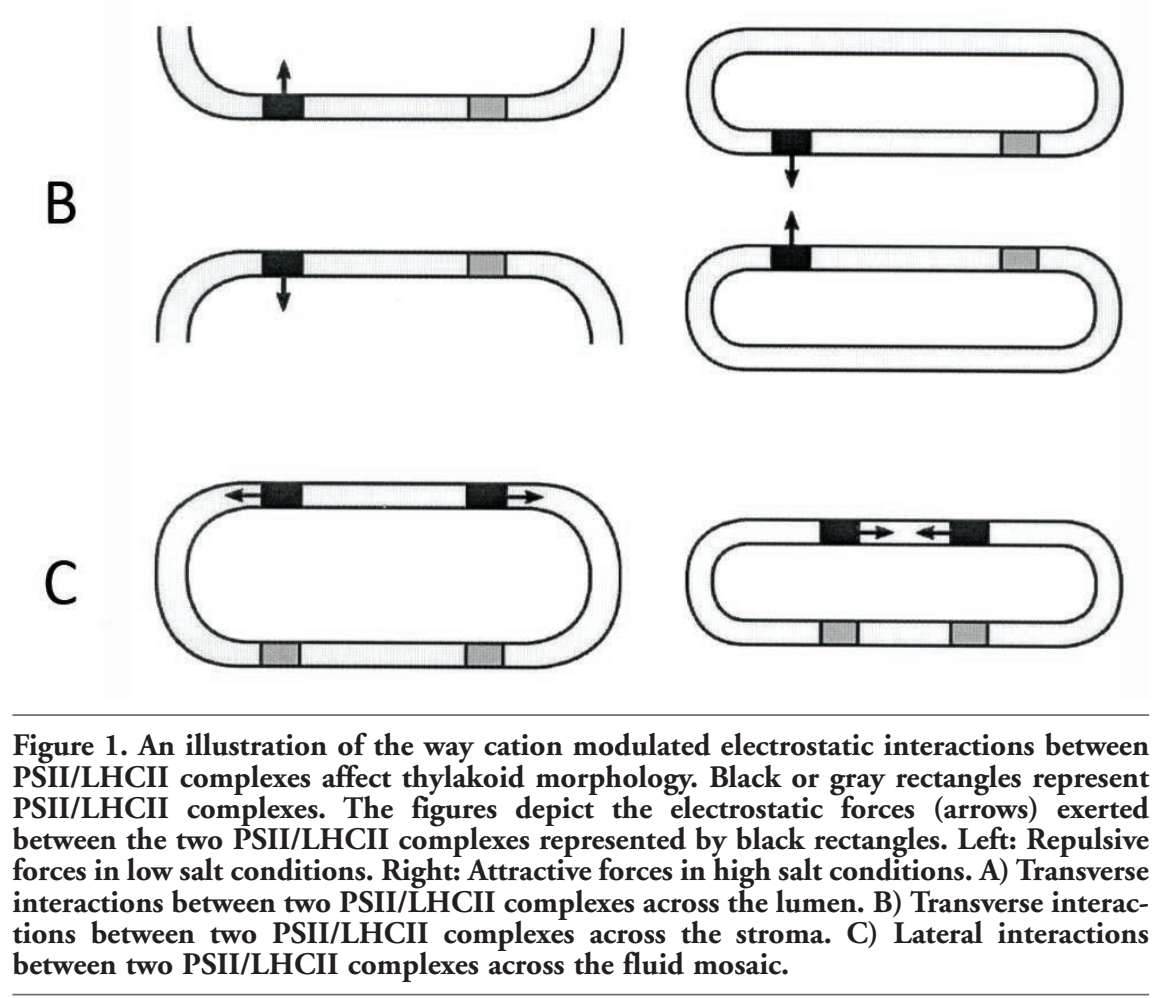
(n) . 
greater extent of stacking was mainly attributed to a greater contribution to Van der Waals attraction, due to the increased amount of PSII light harvesting complexes. It was also attributed to the lateral segregation of PSI into the non-appressed membrane domains, because of the excess of negative charges it carries on the stroma-facing side of the membrane. ${ }^{10}$ According to the rotational model the greater extent of stacking can be explained by the presence of greater amounts of LHCII complexes, thus the distances between the complexes diminish and the attractive forces become strong enough to cause the migration of the complexes and the formation of aggregates and stacks by rotation.

When the chlorophyll-b-deficient CD3 mutants of wheat are grown under low to moderate light, they exhibit significant amount of stacking with more extensive arrays of parallel stroma thylakoids. Under high light conditions, very few membrane stacks were produced, with most of the thylakoids organized in long parallel membrane sheets. ${ }^{26}$ According to the present model, thylakoids in high light have a parallel configuration, because the amount of PSII/LHCII complexes is not sufficient to provoke their lateral aggregation. On the contrary, when grown in low light, the number of PSI/LHCII complexes exceeds the required threshold and they migrate laterally, they form aggregates and finally, they rotate to generate stacks.

Izawa and $\operatorname{Good}^{5}$ found out that spinach chloroplasts isolated in low-salt media lose their grana and look considerably swollen; upon addition of cations, grana reappear and the chloroplasts shrink. Similar observations have been reported with intact bean and pea chloroplasts that were incubated with increasing concentrations of $\mathrm{MgCl}_{2}$ and examined with confocal laser scanning microscopy. ${ }^{27}$ It has also been noticed that in leaf samples of illuminated plants, chloroplasts have an ellipsoidal shape and their thylakoids show the well-known differentiation into unstacked stroma and stacked grana thylakoid membranes. In contrast, in leaf samples of plants after 3 or 7,5 hours of darkness, grana stacks are disintegrated to a large extent and chloroplasts look swollen. ${ }^{28}$ If grana were vertical cylindrical stacks of membranous disks, one would expect the opposite to occur: in the presence of grana chloroplasts would have a swollen shape, while in their absence an ellipsoidal one. The present model denies the existence of cylindrical piles of membranous discs and gives a logical explanation to the different configurations of chloroplasts (Figures 2A,B).

A prerequisite for thylakoid stacking models is membrane continuity enclosing a single lumenal space. This model suggests that, during the rotation of thylakoids, disruptions may occur especially when unstacked thylakoids are directly converted into stacked by addition of cations in vitro. As already mentioned, such disruptions have been reported very early by Izawa and Good. ${ }^{5}$ However, this model advocates that in vivo, the fission of thylakoids occurring during the rotation may be accompa- nied by subsequent fusion of the membranes, which thus become continuous. The presence of a protein that mediates fusion and affects thylakoid organization in Arabidopsis chloroplasts strongly supports this admission. ${ }^{24}$
A
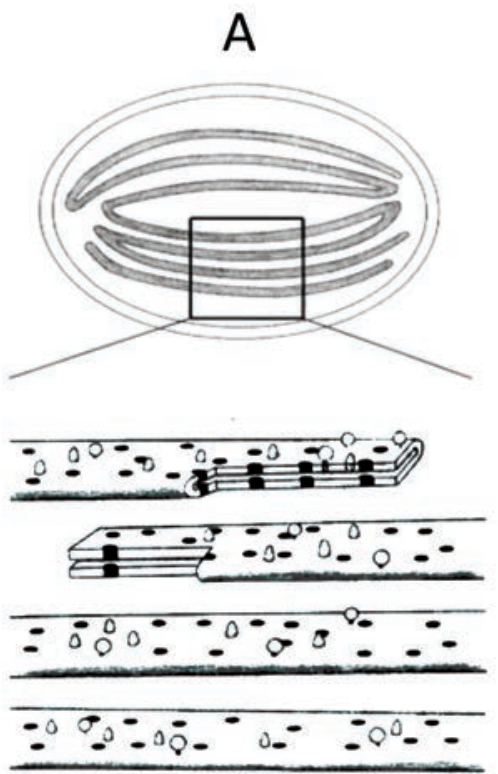

Step 1

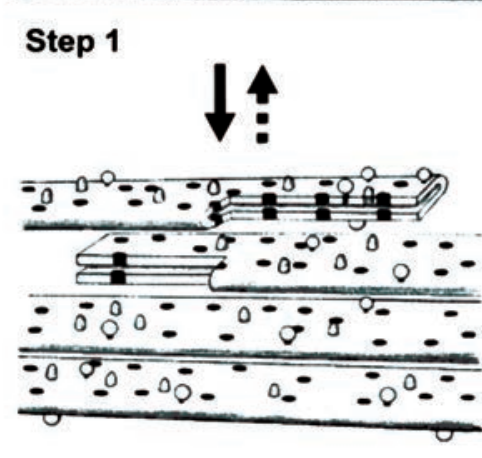

\section{Step 2}

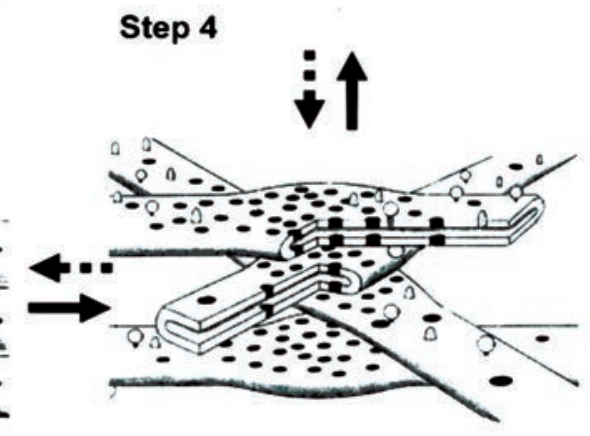

Figure 2. The principle of the rotational model: Steps 1-4 represent zoomed, enlarged, three-dimensional regions of the rectangles in A) and B). The two upper thylakoids of steps 1-4 are sectioned transversely so the lumen, its width and the internal superimposed complexes are visible. The depictions of the complexes and of the membranes are schematic and not in scale. Black discs: PSII/LHCII complexes. Cones: PSI/LHCI complexes. Spheres on cylinders: ATP synthase. Thylakoid stacking (continuous arrows): A) A swollen chloroplast in low salt conditions is suspended in a high salt buffer and attractive electrostatic forces are exerted. Step 1: The adjacent membranes are arranged in a parallel way. Step 2: The membranes come even closer forming bunches of parallel thylakoids. Step 3: The neighboring PSII/LHCII complexes migrate laterally forming circular aggregates, due to attractive electrostatic forces. The thylakoids begin to rotate around the vertical axis of the aggregates. Step 4: Four thylakoids in close contact forming a stack by rotation. B) An ellipsoidal chloroplast in high salt conditions. The rectangle represents a vertical cross section of the stack in step 4. Thylakoid unstacking (dotted arrows): B) An ellipsoidal chloroplast in high salt conditions is suspended in a low salt buffer and repulsive electrostatic forces are exerted. Step 4: Four thylakoids are initially in close contact forming a stack. Step 3: The PSII/LHCII complexes become negatively charged and segregate laterally, due to repulsive forces. The thylakoids begin to move apart. Step 2: The membranes form bunches of parallel thylakoids. Step 1: The distance between the parallel membranes increases. A) A swollen chloroplast in low salt conditions. The rectangle represents a vertical cross section of thylakoids in step 1. 


\section{Conclusions}

The first models on thylakoid stacking appeared over 50 years ago, at the beginning of the 1960s. Ever since, other models have been presented, all of which emphasizing the cylindrical form of the granum. They also satisfy the requirement of membrane continuity, enclosing a single luminal space. ${ }^{3}$ However, none of these models can explain the mechanism of their rapid self-assembly and disassembly on a molecular basis, upon the influence of the physicochemical forces and the involvement of LHCII. None of them can provide proof for the evolutionary origin of these cylindrical structures and their ontogenetic continuity.

The rotational model rejects the generally accepted idea that grana are cylindrical structures and also suggests that thylakoid stacking is the consequence of the lateral heterogeneity and not the inverse. So far, this model is the only one, which provides logical explanations to the rapid response to light - shade conditions, to the changes in the shape of chloroplasts, offering a dynamic and reversible mechanism for thylakoid stacking. Furthermore, it is the only model that implicates the role of certain physicochemical forces and explains the appearance of different conformations of thylakoid membranes that are observed during the evolution or photomorphogenesis of chloroplasts (parallel thylakoids, formation of bunches, appearance of stacks). At the same time it is consistent with many experimental findings.

All things considered, the solution of the granum enigma is of great importance because the numerous functions that are attributed to thylakoids are closely correlated with the mechanism of their self-assembly and disassembly.

\section{References}

1. Anderson JM, Chow WS, De Las Rivas J. Dynamic flexibility in the structure and function of photosystem II in higher plant thylakoid membranes, the grana enigma. Photosynth Res 2008;98:575-87.

2. Dekker JP, Boekema EG. Supramolecular organization of thylakoid membrane proteins in green plants. Biochim Biophys Acta 2005;1706:12-39.

3. Mustardy L, Garab G. Granum revisited. A three-dimensional model - where things fall into place. Trends Plant Sci 2003;8:11722.
4. Boardman NK. Comparative photosynthesis of sun and shade plants. Annu Rev Plant Physiol Plant Mol Biol 1977;28:355-77.

5. Izawa S, Good NE. Effect of salts and electron transport on the conformation of isolated chloroplasts. II. Electron Microscopy. Plant Physiol 1966;41:544-52.

6. Rozak PR, Seiser RM, Wacholtz RM, Wise RR. Rapid, reversible alterations in spinach thylakoid appression upon changes in light intensity. Plant Cell Environ 2002;25:421-9.

7. Chow WS, Kim EH, Horton P, Anderson JM. Granal stacking of thylakoid membranes in higher plant chloroplasts: the physicochemical forces at work and the functional consequences that ensue. Photochem Photobiol Sci 2005;4:1081-90.

8. Argyroudi-Akoyunoglou JH, Feleki Z, Akoyunoglou G. Formation of two chlorophyll-protein complexes during greening of etiolated bean leaves. Biochem Biophys Res Commun 1971;45:606-14.

9. Argyroudi-Akoyunoglou JH, Tsakiris S. Development of the cation-induced stacking capacity during the biogenesis of higher plant thylakoids. Arch Biochem Biophys 1977;184:307-15.

10. Chow WS, Miller S, Anderson JM. Surface charges, the heterogeneous lateral distribution of the two photosystems and thylakoid stacking. Biochim Biophys Acta 1991;1057:69-77.

11. Standfuss J, Van Scheltinga TAC, Lamborgini M Kuhlbrandt W. Mechanisms of photoprotection and non photochemical quenching in pea light harvesting complex at 2,5A resolution. EMBO J 2005;24:919-28.

12. Nevo R, Chuartzman SG, Tsabari 0, et al. Architecture of thylakoid membrane networks. In: H. Wada and N Murata eds. Lipids in photosynthesis: essential and regulatory functions. Springer Science + Business Media B.V; 2009. pp. 295-328.

13. Arvidsson PO, Sundby C. A model for the topology of the chloroplast thylakoid membrane. Aust J Plant Physiol 1999;26:687-94.

14. Chuartzman SG, Nevo R, Shimoni E, et al. Thylakoid membrane remodeling during state transitions in arabidopsis. Plant Cell 2008;20:1029-39.

15. Shimoni E, Rav-Hon 0, Ohad I, et al. Three-dimensional organization of higher-plant chloroplast thylakoid membranes revealed by electron tomography. Plant Cell 2005;17:2580-6.

16. Allen JF, Vermaas WFJ. Evolution of photosynthesis. In: Encyclopedia of Life Sciences (ELS). John Wiley \& Sons eds. Ltd: Chichester. 2010. pp 1-11.
17. Mullineaux CW. Function and evolution of grana. Trends Plant Sci 2005;10:521-5.

18. Wellburn FAM, Wellburn AR. Developmental changes occurring in isolated intact etioplasts. J Cell Sci 1971;9: 271-87.

19. Akoyunoglou G. Biogenesis of photosynthetic membranes in higher plants. In: Bioenergetics of membranes. L. Packer GC, Papageorgiou, Trebst A, eds. Elsevier/ North-Holland Biomedical Press. 1977.

20. Akoyunoglou G, Argyroudi-Akoyunoglou JH, Christias C, et al. Thylakoid Growth and Differentiation in continuous Light as controlled by the duration of preexposure to periodic Light. In: G. Akoyounoglou and J.H. Argyroudi - Akoyounoglou eds. Chloroplast Development. Elsevier/NorthHolland Biomedical Press. 1978. pp 843-56.

21. Barber J. Membrane surface charges and potentials in relation to photosynthesis. Biochim Biophys Acta 1980;594:253-308.

22. Kohorn BD, Yakir D. Movement of newly imported light-harvesting chlorophyllbinding protein from unstacked to stacked thylakoid membranes is not affected by light treatment or absence of amino-terminal threonines. J Biol Chem 1990;265: 2118-23.

23. Kouril R, Oostergetel GT, Boekema EJ. Fine structure of thylakoid membrane organization using cryo electron tomography. Biochim Biophys Acta 2011;1807:36874.

24. Gao H, Sage TL, Osteryoung KW. FZL, an FZO-like protein in plants, is a determinant of thylakoid and chloroplast morphology. Proc. National Academy of Sciences of the United States of America (PNAS). 2006;103:6759-64.

25. Horton P, Ruban AV, Rees D, et al. Control of the light-harvesting function of chloroplast membrane by aggregation of the LHCII chlorophyll-protein complex. FEBS Lett 1991;292:1-4.

26. Falbel TG, Meel JB, Staehelin A. Severity of mutant phenotype in a series of chlorophyll-deficient wheat mutants depends in light intensity and the severity of the block in chlorophyll synthesis. Plant Physiol 1996;112:821-32.

27. Rumak I, Gieczewska K, Kierdaszuk B, et al. 3-D modelling of chloroplast structure under $(\mathrm{Mg} 2+)$ magnesium ion treatment. Relationship between thylakoid membrane arrangement and stacking. Biochim Biophys Acta 2010;1797:1736-48.

28. Pfeiffer S, Krupinska K. New insights in thylakoid membrane organization. Plant Cell Physiol 2005;46:1443-51. 